\title{
Implementation of intimate partner violence education for adolescents with spina bifida
}

\author{
Tal Levin-Decanini ${ }^{\mathrm{a}, \mathrm{b}}$, Elizabeth Miller ${ }^{\mathrm{c}}$, Amy Houtrow ${ }^{\mathrm{d}}$, Lisa Kreashko ${ }^{\mathrm{a}}$ and Brenda Cassidy ${ }^{\mathrm{a}, *}$ \\ ${ }^{a}$ University of Pittsburgh School of Nursing, University of Pittsburgh, Pittsburgh, PA, USA \\ ${ }^{\mathrm{b}}$ Children's Hospital of Pittsburgh of UPMC, Pittsburgh, PA, USA \\ ${ }^{\mathrm{c}}$ Division of Adolescent and Young Adult Medicine, University of Pittsburgh School of Medicine, Children's \\ Hospital of Pittsburgh of UPMC, Pittsburgh, PA, USA \\ ${ }^{\mathrm{d}}$ Department of Physical Medicine and Rehabilitation, University of Pittsburgh School of Medicine, Children's \\ Hospital of Pittsburgh of UPMC, Pittsburgh, PA, USA
}

\begin{abstract}
.
OBJECTIVE: Intimate partner violence (IPV) prevention among adolescent patients with disability is needed, yet rarely discussed in the clinical setting. This study evaluated the feasibility of implementing a brief educational training based on an evidence-based IPV intervention in a pediatric spina bifida clinic. Frequency of IPV discussion was assessed through evaluation of patient feedback and provider surveys.

METHODS: Adolescent patients with spina bifida aged 12-21 completed after visit surveys before $(N=13)$ and after the provider training $(N=21)$. Primary outcomes included frequency of provider discussion about IPV and receipt of patient safety cards. Chi-square tests compared patient feedback prior to and two months following the education session. Provider knowledge and attitude changes were assessed with pre-post surveys.

RESULTS: More patients discussed IPV with providers following the education session compared to baseline $(p=0.03)$. Provider feedback, both immediately and at two months after the education session showed increased awareness of IPV, comfort with assessment, disclosure, and referral to resources.

CONCLUSIONS: The educational intervention increased provider comfort with addressing IPV within a specialty clinical setting. The frequency of IPV communication significantly increased as compared to baseline, patients reported the discussions were beneficial, and providers reported greater comfort discussing IPV and referring patients to resources.
\end{abstract}

Keywords: Spina bifida, adolescent, intimate partner violence

\section{Introduction}

Spina bifida, a common neurodevelopmental disorder, is associated with a broad range of disabilities. Emerging literature indicates that among individuals with spina bifida, adolescents are at high risk for abuse with $37 \%$ of adolescent and young adult females stating they have experienced unwanted sexual attention,

\footnotetext{
${ }^{*}$ Corresponding author: Brenda Cassidy, University of Pittsburgh School of Nursing, Victoria Building, 3500 Victoria Street, Pittsburgh, PA 15261, USA. Tel.: +1 412648 9253; E-mail: cassb@pitt. edu.
}

and 30\% reporting unwanted sexual touching [1]. Intimate partner violence (IPV) includes physical and sexual violence, as well as stalking and psychological manipulation perpetrated by a dating or sexual partner and is not uncommon during adolescence [2]. While a significant health concern for all adolescents, IPV is more prevalent among adolescents and young adults with disabilities $[3,4]$ and is associated with poor health outcomes [3].

Given the high rates of IPV among adolescents with disabilities, identifying strategies to increase awareness of healthy and unhealthy relationships is needed for this vulnerable population. The clinical encounter may be a particularly salient setting for discussing this 
sensitive topic. In this study, we evaluated the feasibility of implementing a previously successful intervention for IPV education in school health centers [5] in a specialty clinic for individuals with spina bifida. Feedback from patient and provider surveys was used to evaluate the acceptability of a tailored IPV education session and whether patient discussion of IPV with their providers increased.

\section{Methods}

\subsection{Design}

Materials and methods for this quality improvement study were adapted from an adolescent IPV educational training program created for adolescent health settings [5] utilizing the Futures Without Violence training materials [6]. For one month prior to an educational training session, baseline data were collected from patients after their clinic visit about whether they discussed IPV during that visit. Following this, all interested Physical Medicine and Rehabilitation (PM\&R) staff $(n=8)$ attended an educational training session based on this previously tested, publicly available training. Attendees completed pre and posttraining questionnaires. The training session reviewed the use of an educational safety card to provide patients with information regarding both IPV and referral resources [2]. For two months following the training, adolescent patients were asked to complete a brief survey about their visit. Providers and staff also completed a follow-up survey two months after the training. University of Pittsburgh Institutional Review Board approval was obtained for this study, which included a waiver of parental permission.

\subsection{Sample and setting}

The spina bifida clinic at Children's Hospital of Pittsburgh of the University of Pittsburgh Medical Center is a specialized clinic staffed by a multidisciplinary health care team. The clinic staff was consistent during the entirety of this QI project, and was comprised of nurses, medical students, trainees, and attending physicians. All English-speaking patients between the ages of 12-21 were eligible to complete the patient feedback surveys and no demographic information was obtained.

\subsection{Procedures}

Education session materials were adapted from $\mathrm{Fu}$ tures Without Violence clinical guidelines on IPV among adolescents [6], and were modified to include information regarding IPV in individuals with disabilities, in particular those with spina bifida. Surveys for providers were modified from previous IPV studies and assessed their confidence with and frequency of discussing IPV during clinic visits. Patient feedback surveys, modified from previous studies [5,7], were completed anonymously after clinical encounters, both prior to the education session to establish a baseline, as well as for two months after the education session. Patients were given their survey during their clinic visit and were instructed to complete it at the completion of their visit. No patient completed the survey more than once. All data collected were anonymous and surveys were stored in a locked box.

Data were analyzed using SPSS. In addition to descriptive statistics for clinician surveys, changes from before and after training in patient feedback were assessed using descriptive statistics, Fisher's Exact Tests (analysis of safety card distribution frequency), or Chi-square tests. Responses to patient surveys were compared from pre-training to one-month post, twomonths post, as well as across the two months following the training.

\section{Results}

\subsection{Patient surveys}

For baseline data there were 15 eligible adolescent patients, of which $13(87 \%)$ completed the surveys. Fifteen percent of these patients responded that providers discussed healthy/unhealthy relationships with them, and $31 \%$ stated providers reviewed confidentiality with them (Table 1). About one third stated that discussing relationships was somewhat or very helpful.

During the first month following the education session, patient safety cards were placed among other education materials provided in the clinic. Of the 16 eligible adolescent patients who presented to clinic, eight completed surveys (50\%). Patient reports indicated that the frequency of discussion of healthy/unhealthy relationships had significantly increased with $88 \%$ of respondents stating that such conversations occurred ( $p=0.005$, Table 1$)$, although no safety cards were distributed. There was no significant difference between this time point and baseline regarding discussion of confidentiality $(p>0.05)$.

Thirteen of the 18 adolescent patients seen in clinic during the second month returned surveys $(72 \%)$. To 
Table 1

Patient feedback survey responses noting frequency with which clinic providers discussed healthy/unhealthy relationships, confidentiality, and gave patients the safety card prior to, one month after, and two months after the IPV training session

\begin{tabular}{|c|c|c|c|c|}
\hline Frequency of provider discussion of: & $\begin{array}{c}\text { Baseline (\%) } \\
\quad N=13\end{array}$ & $\begin{array}{c}1 \text { month PT }(\%) \\
N=8\end{array}$ & $\begin{array}{c}2 \text { months PT (\%) } \\
N=13\end{array}$ & $p$ \\
\hline Healthy/unhealthy relationships & 15 & 88 & 54 & $\begin{array}{l}A^{\mathrm{C}}: 0.03 \\
B^{\mathrm{C}}: 0.005 \\
C^{\mathrm{C}}, d^{\mathrm{C}}:>0.05\end{array}$ \\
\hline Confidentiality & 31 & 50 & 39 & $A-D^{\mathrm{C}}>0.05$ \\
\hline Safety card given during visit & 0 & 0 & 62 & $\begin{array}{l}A^{\mathrm{F}}: 0.019 \\
B^{\mathrm{F}}:>0.05 \\
C^{\mathrm{F}}, d^{\mathrm{C}}: \leqslant 0.005\end{array}$ \\
\hline
\end{tabular}

PT, post-training; ${ }^{\mathrm{C}}$ Chi-square; ${ }^{\mathrm{F}}$ Fisher's Exact Test; $A$ baseline vs PT; $B$ baseline vs 1 Month PT; $C$ baseline vs 2 Months PT; $D 1$ month PT vs 2 Months PT.

Table 2

Pre-training provider $(N=6)$ survey responses of the frequency with which they discussed DSV, confidentiality, and assessed the safety of their adolescent patients

\begin{tabular}{lccccc}
\hline & \multicolumn{5}{c}{ Frequency of discussion } \\
\cline { 2 - 6 } & $\begin{array}{c}\text { All of } \\
\text { the time } \\
(\%)\end{array}$ & $\begin{array}{c}\text { Most of } \\
\text { the time } \\
(\%)\end{array}$ & $\begin{array}{c}\text { Some of } \\
\text { the time } \\
(\%)\end{array}$ & $\begin{array}{c}\text { Not so } \\
\text { often } \\
(\%)\end{array}$ & $\begin{array}{c}\text { Rarely } \\
(\%)\end{array}$ \\
\hline DSV & 0 & 0 & 17 & 17 & 67 \\
Confidentiality & 17 & 17 & 0 & 17 & 33 \\
Assess safety & 0 & 0 & 17 & 17 & 67 \\
\hline
\end{tabular}

DSV: domestic and sexual violence.

Table 3

Provider self-assessment survey $(N=4)$

\begin{tabular}{lccccc}
\hline & \multicolumn{5}{c}{ Frequency of discussion } \\
\cline { 2 - 6 } & $\begin{array}{c}\text { All of } \\
\text { the time } \\
(\%)\end{array}$ & $\begin{array}{c}\text { Most of } \\
\text { the time } \\
(\%)\end{array}$ & $\begin{array}{c}\text { Some of } \\
\text { the time } \\
(\%)\end{array}$ & $\begin{array}{c}\text { Not so } \\
\text { often } \\
(\%)\end{array}$ & $\begin{array}{c}\text { Rarely } \\
(\%)\end{array}$ \\
\hline DSV & 0 & 25 & 0 & 25 & 50 \\
Confidentiality & 50 & 0 & 25 & 0 & 25 \\
Assess safety & 0 & 25 & 0 & 25 & 50 \\
\hline
\end{tabular}

DSV: domestic and sexual violence.

remind providers to distribute the patient safety cards, these were attached to the charts of all eligible patients. This increased the use of the cards from $0 \%$ to $62 \%$ of survey respondents $(p=0.005)$. In the second month, $88 \%$ of respondents stated this increased their understanding of how to help someone affected by IPV. However, rate of discussion of healthy/unhealthy relationships fell to 54\%, which was not significantly different from baseline $(p>0.05)$ or the first month $(p>$ 0.05).

Following the education session, the frequency of discussion about healthy/unhealthy relationships over the next two months increased significantly ( $p=0.03$, Table 1). There was no significant difference across the time points related to frequency of discussion of confidentiality. A majority of patients who received the informational cards found them to be useful, although to promote provider discussion of the cards these had to be prominently placed.

\subsection{Provider training}

The education session was attended by eight spina bifida clinic staff during a departmental meeting: an administrator, two nurses, and five physicians. Six of the attendees completed pre-education surveys, and all eight attendees completed a post-training survey immediately after the training session. In the pre-training survey, for questions regarding frequency of discussion of IPV, confidentiality as well as assessing a patient's safety in a relationship, most individuals stated they had such discussions "not so often" or "rarely" (see Table 2). The most often cited reasons for not addressing such issues included not having enough time, not being sure how to ask, and only asking if the patient raises the concern. Of the six providers, one suggested that prompts in the electronic record would be helpful. Five noted that the clinic had no materials related to IPV or reproductive coercion.

All respondents to the post-training either agreed or strongly agreed with the following statements: the education session was effective in increasing understanding of domestic and sexual violence, discussion of confidentiality with patients, ways to provide education, and how to obtain access to resources. In addition, all respondents either agreed or strongly agreed that they would be more likely to provide education regarding IPV and to discuss confidentiality. Two months later, four physicians in the spina bifida clinic completed a self-assessment survey. Most reported that they discussed IPV and confidentiality, and were more likely to assess safety of their patients. All respondents either agreed or strongly agreed that they were more comfortable responding to clients who disclose abuse and were either somewhat or completely confident in referring a patient to a partner IPV organization (Table 3). 


\section{Discussion}

Overall the results of this quality improvement study support the feasibility of adapting this universal education intervention for specialty clinics serving more vulnerable adolescents. The adapted IPV intervention increased discussion between providers and patients about relationships and IPV. In addition, providers noted increased comfort with the subject matter, how to respond to disclosure, and referring individuals to partner organizations. Distribution of the safety card was limited (40\% adoption), and even prompts like placing the cards on the patient's chart did not achieve $100 \%$ adoption.

The findings from this implementation are encouraging, particularly that with minimal modifications the educational content clearly resonated with the young people. However, care must be taken not to overgeneralize given the study limitations, including small provider as well as patient samples, and inclusion of only including English speaking adolescents. Although all eligible individuals were informed that completion of the survey was voluntary and no demographic patient information was being recorded, patients may have felt uncomfortable completing the survey. In addition, parents were frequently in the room with the patient which may have limited both provider discussion as well as willingness of the adolescent to respond to the questionnaire. Patient data, based on their recollection of the interaction with the providers, is subject to recall bias. Nonetheless, this study indicates that an intervention consisting of one education session and patient review of the safety card can increase provider knowledge and comfort discussing IPV, and also increases patient awareness of IPV and relevant resources.

Adolescents and young adults with disabilities are significantly more likely to experience IPV than their peers without disabilities [3,8-10]. Emerging adults with disabilities often do not receive information beyond general school-based sexual education, leaving significant gaps in their knowledge of how disability may affect sexual function, as well as information regarding dating violence identification and prevention. Prior studies have shown the need for increased provider-based education on these topics [1,11-13], as well as improved screening for IPV [14]. This evidence-based intervention meets the need of this vulnerable population. In this quality improvement study, a previously successful intervention for IPV education was successfully modified and implemented for use in a specialty pediatric clinic for individuals with spina bifida. Providing universal access to information regarding healthy/unhealthy relationships to adolescents and young adults with disabilities supports and normalizes the role of these individuals as active participants in the community who have the power to help their friends with this information.

\section{Conflict of interest}

The authors have no conflict of interest to report.

\section{References}

[1] Sawyer SM, Roberts KV. Sexual and reproductive health in young people with spina bifida. Dev Med Child Neurol. 1999; 41(10): 671-5.

[2] Miller E. Prevention of and interventions for dating and sexual violence in adolescence. Pediatr Clin North Am. 2017; 64(2): 423-34.

[3] Mitra M, Mouradian VE, McKenna M. Dating violence and associated health risks among high school students with disabilities. Matern Child Health J. 2013; 17(6): 1088-94.

[4] Alriksson-Schmidt AI, Thibadeau JK, Swanson ME, Marcus D, Carris KL, Siffel C, et al. The natural history of spina bifida in children pilot project: Research protocol. J Med Internet Res. 2013; 15(1).

[5] Miller E, Goldstein S, McCauley HL, Jones KA, Dick RN, Jetton J, et al. A school health center intervention for abusive adolescent relationships: A cluster RCT. Pediatrics. 2015; 135(1): 76-85.

[6] Miller E, Levenson R. Hanging out or hooking up: Clinical guidelines on responding to adolescent relationship abuse futures without violence. 2013. [Available from: https://www. futureswithoutviolence.org/userfiles/file/HealthCare/Adoles cent $\% 20$ Health\%20Guide.pdf.

[7] Tancredi DJ, Silverman JG, Decker MR, McCauley HL, Anderson HA, Jones KA, et al. Cluster randomized controlled trial protocol: Addressing reproductive coercion in health settings (ARCHES). BMC Womens Health. 2015; 15: 57.

[8] Alriksson-Schmidt AI, Armour BS, Thibadeau JK. Are adolescent girls with a physical disability at increased risk for sexual violence? J School Health. 2010; 80(7): 361-7.

[9] Scherer HL, Snyder JA, Fisher BS. Intimate partner victimization among college students with and without disabilities: Prevalence of and relationship to emotional well-being. Journal of Interpersonal Violence. 2016; 31(1): 49-80.

[10] Basile KC, Breiding MJ, Smith SG. Disability and risk of recent sexual violence in the United States. American Journal of Public Health. 2016; 106(5): 928-33.

[11] Cardenas DA, Topolski TA, White CJ, McLaughlin JF, Walker WO. Sexual functioning in adolescents and young adults with spina bifida. Arch Phys Med Rehab. 2008; 89(1): 31-5.

[12] Verhoef M, Barf HA, Vroege JA, Post MW, Van Asbeck FW, Gooskens RH, et al. Sex education, relationships, and sexuality in young adults with spina bifida. Arch Phys Med Rehabil. 2005; 86(5): 979-87. 
[13] Akre C, Light A, Sherman L, Polvinen J, Rich M. What young people with spina bifida want to know about sex and are not being told. Child Care Health Dev. 2015; 41(6): 963-9.
[14] Plummer SB, Findley PA. Women with disabilities' experience with physical and sexual abuse: Review of the literature and implications for the field. Trauma Violence Abuse. 2012; 13(1): 15-29. 